DESY-98-089

\title{
Measurement of Elastic $\Upsilon$ Photoproduction at HERA
}

\section{ZEUS Collaboration}

\begin{abstract}
The photoproduction reaction $\gamma p \rightarrow \mu^{+} \mu^{-} p$ has been studied in $e p$ interactions using the ZEUS detector at HERA. The data sample corresponds to an integrated luminosity of $43.2 \mathrm{pb}^{-1}$. The $\Upsilon$ meson has been observed in photoproduction for the first time. The sum of the products of the elastic $\Upsilon(1 S), \Upsilon(2 S), \Upsilon(3 S)$ photoproduction cross sections with their respective branching ratios is determined to be $13.3 \pm 6.0(\text { stat. })_{-2.3}^{+2.7}$ (syst.) pb at a mean photon-proton centre of mass energy of $120 \mathrm{GeV}$. The cross section is above the prediction of a perturbative QCD model.
\end{abstract}




\section{The ZEUS Collaboration}

J. Breitweg, M. Derrick, D. Krakauer, S. Magill, D. Mikunas, B. Musgrave, J. Repond, R. Stanek, R.L. Talaga, R. Yoshida, H. Zhang

Argonne National Laboratory, Argonne, IL, USA ${ }^{p}$

M.C.K. Mattingly

Andrews University, Berrien Springs, MI, USA

F. Anselmo, P. Antonioli, G. Bari, M. Basile, L. Bellagamba, D. Boscherini, A. Bruni, G. Bruni, G. Cara Romeo, G. Castellini ${ }^{1}$, L. Cifarelli ${ }^{2}$, F. Cindolo, A. Contin, N. Coppola, M. Corradi, S. De Pasquale, P. Giusti, G. Iacobucci, G. Laurenti, G. Levi, A. Margotti, T. Massam, R. Nania, F. Palmonari, A. Pesci, A. Polini, G. Sartorelli, Y. Zamora Garcia ${ }^{3}$, A. Zichichi

University and INFN Bologna, Bologna, Italy ${ }^{f}$

C. Amelung, A. Bornheim, I. Brock, K. Coböken, J. Crittenden, R. Deffner, M. Eckert, M. Grothe ${ }^{4}$, H. Hartmann, K. Heinloth, L. Heinz, E. Hilger, H.-P. Jakob, A. Kappes, U.F. Katz, R. Kerger, E. Paul, M. Pfeiffer, H. Schnurbusch, H. Wieber

Physikalisches Institut der Universität Bonn, Bonn, Germany ${ }^{c}$

D.S. Bailey, S. Campbell-Robson, W.N. Cottingham, B. Foster, R. Hall-Wilton, G.P. Heath, H.F. Heath, J.D. McFall, D. Piccioni, D.G. Roff, R.J. Tapper

H.H. Wills Physics Laboratory, University of Bristol, Bristol, U.K. ${ }^{o}$

M. Capua, L. Iannotti, A. Mastroberardino, M. Schioppa, G. Susinno

Calabria University, Physics Dept.and INFN, Cosenza, Italy ${ }^{f}$

J.Y. Kim, J.H. Lee, I.T. Lim, M.Y. Pac ${ }^{5}$

Chonnam National University, Kwangju, Korea ${ }^{h}$

A. Caldwell ${ }^{6}$, N. Cartiglia, Z. Jing, W. Liu, B. Mellado, J.A. Parsons, S. Ritz ${ }^{7}$, S. Sampson, F. Sciulli, P.B. Straub, Q. Zhu

Columbia University, Nevis Labs., Irvington on Hudson, N.Y., USA ${ }^{q}$

P. Borzemski, J. Chwastowski, A. Eskreys, J. Figiel, K. Klimek, M.B. Przybycień, L. Zawiejski

Inst. of Nuclear Physics, Cracow, Poland ${ }^{j}$

L. Adamczyk ${ }^{8}$, B. Bednarek, M. Bukowy, A.M. Czermak, K. Jeleń, D. Kisielewska, T. Kowalski,

M. Przybycień, E. Rulikowska-Zarȩbska, L. Suszycki, J. Zajạc

Faculty of Physics and Nuclear Techniques, Academy of Mining and Metallurgy, Cracow, Poland $^{j}$

Z. Duliński, A. Kotański

Jagellonian Univ., Dept. of Physics, Cracow, Poland ${ }^{k}$ 
G. Abbiendi ${ }^{9}$, L.A.T. Bauerdick, U. Behrens, H. Beier ${ }^{10}$, J.K. Bienlein, K. Desler, G. Drews, U. Fricke, I. Gialas ${ }^{11}$, F. Goebel, P. Göttlicher, R. Graciani, T. Haas, W. Hain, G.F. Hartner, D. Hasell ${ }^{12}$, K. Hebbel, K.F. Johnson ${ }^{13}$, M. Kasemann, W. Koch, U. Kötz, H. Kowalski, L. Lindemann, B. Löhr, M. Martínez, J. Milewski, M. Milite, T. Monteiro ${ }^{14}$, D. Notz, A. Pellegrino, F. Pelucchi, K. Piotrzkowski, M. Rohde, J. Roldán ${ }^{15}$, J.J. Ryan ${ }^{16}$, P.R.B. Saull, A.A. Savin, U. Schneekloth, O. Schwarzer, F. Selonke, S. Stonjek, B. Surrow ${ }^{17}$, E. Tassi, D. Westphal ${ }^{18}$, G. Wolf, U. Wollmer, C. Youngman, W. Zeuner

Deutsches Elektronen-Synchrotron DESY, Hamburg, Germany

B.D. Burow, C. Coldewey, H.J. Grabosch, A. Meyer, S. Schlenstedt

DESY-IfH Zeuthen, Zeuthen, Germany

G. Barbagli, E. Gallo, P. Pelfer

University and INFN, Florence, Italy ${ }^{f}$

G. Maccarrone, L. Votano

INFN, Laboratori Nazionali di Frascati, Frascati, Italy ${ }^{f}$

A. Bamberger, S. Eisenhardt, P. Markun, H. Raach, T. Trefzger ${ }^{19}$, S. Wölfle

Fakultät für Physik der Universität Freiburg i.Br., Freiburg i.Br., Germany ${ }^{c}$

J.T. Bromley, N.H. Brook, P.J. Bussey, A.T. Doyle ${ }^{20}$, S.W. Lee, N. Macdonald, G.J. McCance, D.H. Saxon, L.E. Sinclair, I.O. Skillicorn, E. Strickland, R. Waugh

Dept. of Physics and Astronomy, University of Glasgow, Glasgow, U.K. ${ }^{o}$

I. Bohnet, N. Gendner, U. Holm, A. Meyer-Larsen, H. Salehi, K. Wick

Hamburg University, I. Institute of Exp. Physics, Hamburg, Germany ${ }^{c}$

A. Garfagnini, L.K. Gladilin ${ }^{21}$, D. Kçira ${ }^{22}$, R. Klanner, E. Lohrmann, G. Poelz, F. Zetsche Hamburg University, II. Institute of Exp. Physics, Hamburg, Germany ${ }^{c}$

T.C. Bacon, I. Butterworth, J.E. Cole, G. Howell, L. Lamberti ${ }^{23}$, K.R. Long, D.B. Miller, N. Pavel, A. Prinias ${ }^{24}$, J.K. Sedgbeer, D. Sideris, R. Walker

Imperial College London, High Energy Nuclear Physics Group, London, U.K. ${ }^{o}$

U. Mallik, S.M. Wang, J.T. $\mathrm{Wu}^{25}$

University of Iowa, Physics and Astronomy Dept., Iowa City, USA ${ }^{p}$

P. Cloth, D. Filges

Forschungszentrum Jülich, Institut für Kernphysik, Jülich, Germany

J.I. Fleck ${ }^{17}$, T. Ishii, M. Kuze, I. Suzuki ${ }^{26}$, K. Tokushuku, S. Yamada, K. Yamauchi, Y. Yamazaki ${ }^{27}$

Institute of Particle and Nuclear Studies, KEK, Tsukuba, Japan ${ }^{g}$

S.J. Hong, S.B. Lee, S.W. Nam ${ }^{28}$, S.K. Park

Korea University, Seoul, Korea ${ }^{h}$

H. Lim, I.H. Park, D. Son

Kyungpook National University, Taegu, Korea ${ }^{h}$

F. Barreiro, J.P. Fernández, G. García, C. Glasman ${ }^{29}$, J.M. Hernández, L. Hervás ${ }^{17}$, L. Labarga, J. del Peso, J. Puga, J. Terrón, J.F. de Trocóniz

Univer. Autónoma Madrid, Depto de Física Teórica, Madrid, Spain ${ }^{n}$ 
F. Corriveau, D.S. Hanna, J. Hartmann, W.N. Murray, A. Ochs, M. Riveline, D.G. Stairs, M. St-Laurent

McGill University, Dept. of Physics, Montréal, Québec, Canada ${ }^{a},{ }^{b}$

T. Tsurugai

Meiji Gakuin University, Faculty of General Education, Yokohama, Japan

V. Bashkirov, B.A. Dolgoshein, A. Stifutkin

Moscow Engineering Physics Institute, Moscow, Russia ${ }^{l}$

G.L. Bashindzhagyan, P.F. Ermolov, Yu.A. Golubkov, L.A. Khein, N.A. Korotkova, I.A. Korzhavina, V.A. Kuzmin, O.Yu. Lukina, A.S. Proskuryakov, L.M. Shcheglova ${ }^{30}$, A.N. Solomin ${ }^{30}$, S.A. Zotkin

Moscow State University, Institute of Nuclear Physics, Moscow, Russia ${ }^{m}$

C. Bokel, M. Botje, N. Brümmer, J. Engelen, E. Koffeman, P. Kooijman, A. van Sighem, H. Tiecke, N. Tuning, W. Verkerke, J. Vossebeld, L. Wiggers, E. de Wolf

NIKHEF and University of Amsterdam, Amsterdam, Netherlands ${ }^{i}$

D. Acosta ${ }^{31}$, B. Bylsma, L.S. Durkin, J. Gilmore, C.M. Ginsburg, C.L. Kim, T.Y. Ling, P. Nylander, T.A. Romanowski ${ }^{32}$

Ohio State University, Physics Department, Columbus, Ohio, USA ${ }^{p}$

H.E. Blaikley, R.J. Cashmore, A.M. Cooper-Sarkar, R.C.E. Devenish, J.K. Edmonds, J. Große-Knetter ${ }^{33}$, N. Harnew, C. Nath, V.A. Noyes ${ }^{34}$, A. Quadt, O. Ruske, J.R. Tickner ${ }^{35}$, R. Walczak, D.S. Waters

Department of Physics, University of Oxford, Oxford, U.K. ${ }^{o}$

A. Bertolin, R. Brugnera, R. Carlin, F. Dal Corso, U. Dosselli, S. Limentani, M. Morandin, M. Posocco, L. Stanco, R. Stroili, C. Voci

Dipartimento di Fisica dell' Università and INFN, Padova, Italy ${ }^{f}$

B.Y. Oh, J.R. Okrasiński, W.S. Toothacker, J.J. Whitmore

Pennsylvania State University, Dept. of Physics, University Park, PA, USA ${ }^{q}$

Y. Iga

Polytechnic University, Sagamihara, Japan ${ }^{g}$

G. D'Agostini, G. Marini, A. Nigro, M. Raso

Dipartimento di Fisica, Univ. 'La Sapienza' and INFN, Rome, Italy ${ }^{f}$

J.C. Hart, N.A. McCubbin, T.P. Shah

Rutherford Appleton Laboratory, Chilton, Didcot, Oxon, U.K. ${ }^{o}$

D. Epperson, C. Heusch, J.T. Rahn, H.F.-W. Sadrozinski, A. Seiden, R. Wichmann, D.C. Williams

University of California, Santa Cruz, CA, USA ${ }^{p}$

H. Abramowicz ${ }^{36}$, G. Briskin ${ }^{37}$, S. Dagan ${ }^{38}$, S. Kananov ${ }^{38}$, A. Levy ${ }^{38}$

Raymond and Beverly Sackler Faculty of Exact Sciences, School of Physics, Tel-Aviv University,

Tel-Aviv, Israel $^{e}$

T. Abe, T. Fusayasu, M. Inuzuka, K. Nagano, K. Umemori, T. Yamashita

Department of Physics, University of Tokyo, Tokyo, Japan ${ }^{g}$ 
R. Hamatsu, T. Hirose, K. Homma ${ }^{39}$, S. Kitamura ${ }^{40}$, T. Matsushita, T. Nishimura Tokyo Metropolitan University, Dept. of Physics, Tokyo, Japan ${ }^{g}$

M. Arneodo ${ }^{20}$, R. Cirio, M. Costa, M.I. Ferrero, S. Maselli, V. Monaco, C. Peroni, M.C. Petrucci, M. Ruspa, R. Sacchi, A. Solano, A. Staiano

Università di Torino, Dipartimento di Fisica Sperimentale and INFN, Torino, Italy ${ }^{f}$

M. Dardo

II Faculty of Sciences, Torino University and INFN - Alessandria, Italy ${ }^{f}$

D.C. Bailey, C.-P. Fagerstroem, R. Galea, K.K. Joo, G.M. Levman, J.F. Martin R.S. Orr, S. Polenz, A. Sabetfakhri, D. Simmons

University of Toronto, Dept. of Physics, Toronto, Ont., Canada ${ }^{a}$

J.M. Butterworth, C.D. Catterall, M.E. Hayes, E.A. Heaphy, T.W. Jones, J.B. Lane, R.L. Saunders, M.R. Sutton, M. Wing

University College London, Physics and Astronomy Dept., London, U.K. ${ }^{o}$

J. Ciborowski, G. Grzelak ${ }^{41}$, R.J. Nowak, J.M. Pawlak, R. Pawlak, B. Smalska,

T. Tymieniecka, A.K. Wróblewski, J.A. Zakrzewski, A.F. Żarnecki

Warsaw University, Institute of Experimental Physics, Warsaw, Poland ${ }^{j}$

M. Adamus

Institute for Nuclear Studies, Warsaw, Poland ${ }^{j}$

O. Deppe, Y. Eisenberg ${ }^{38}$, D. Hochman, U. Karshon ${ }^{38}$

Weizmann Institute, Department of Particle Physics, Rehovot, Israel ${ }^{d}$

W.F. Badgett, D. Chapin, R. Cross, S. Dasu, C. Foudas, R.J. Loveless, S. Mattingly, D.D. Reeder, W.H. Smith, A. Vaiciulis, M. Wodarczyk

University of Wisconsin, Dept. of Physics, Madison, WI, USA ${ }^{p}$

A. Deshpande, S. Dhawan, V.W. Hughes

Yale University, Department of Physics, New Haven, CT, USA ${ }^{p}$

S. Bhadra, W.R. Frisken, M. Khakzad, W.B. Schmidke

York University, Dept. of Physics, North York, Ont., Canada ${ }^{a}$ 
1 also at IROE Florence, Italy

2 now at Univ. of Salerno and INFN Napoli, Italy

${ }^{3}$ supported by Worldlab, Lausanne, Switzerland

${ }^{4}$ now at University of California, Santa Cruz, USA

${ }^{5}$ now at Dongshin University, Naju, Korea

6 also at DESY

7 Alfred P. Sloan Foundation Fellow

${ }^{8}$ supported by the Polish State Committee for Scientific Research, grant No. 2P03B14912

${ }^{9}$ now at INFN Bologna

10 now at Innosoft, Munich, Germany

${ }^{11}$ now at Univ. of Crete, Greece, partially supported by DAAD, Bonn - Kz. A/98/16764

12 now at Massachusetts Institute of Technology, Cambridge, MA, USA

13 visitor from Florida State University

14 supported by European Community Program PRAXIS XXI

15 now at IFIC, Valencia, Spain

16 now a self-employed consultant

17 now at CERN

18 now at Bayer A.G., Leverkusen, Germany

19 now at ATLAS Collaboration, Univ. of Munich

20 also at DESY and Alexander von Humboldt Fellow at University of Hamburg

21 on leave from MSU, supported by the GIF, contract I-0444-176.07/95

22 supported by DAAD, Bonn - Kz. A/98/12712

23 supported by an EC fellowship

${ }^{24}$ PPARC Post-doctoral fellow

25 now at Applied Materials Inc., Santa Clara

26 now at Osaka Univ., Osaka, Japan

27 supported by JSPS Postdoctoral Fellowships for Research Abroad

28 now at Wayne State University, Detroit

29 supported by an EC fellowship number ERBFMBICT 972523

30 partially supported by the Foundation for German-Russian Collaboration DFG-RFBR (grant no. 436 RUS 113/248/3 and no. 436 RUS 113/248/2)

31 now at University of Florida, Gainesville, FL, USA

32 now at Department of Energy, Washington

33 supported by the Feodor Lynen Program of the Alexander von Humboldt foundation

34 Glasstone Fellow

35 now at CSIRO, Lucas Heights, Sydney, Australia

36 an Alexander von Humboldt Fellow at University of Hamburg

37 now at Brown University, Providence, RI, USA

38 supported by a MINERVA Fellowship

39 now at ICEPP, Univ. of Tokyo, Tokyo, Japan

40 present address: Tokyo Metropolitan College of Allied Medical Sciences, Tokyo 116, Japan

${ }^{41}$ supported by the Polish State Committee for Scientific Research, grant No. 2P03B09308 
a supported by the Natural Sciences and Engineering Research Council of Canada (NSERC)

$b$ supported by the FCAR of Québec, Canada

$c$ supported by the German Federal Ministry for Education and Science, Research and Technology (BMBF), under contract numbers 057BN19P, 057FR19P, 057HH19P, 057HH29P

$d$ supported by the MINERVA Gesellschaft für Forschung GmbH, the German Israeli Foundation, the U.S.-Israel Binational Science Foundation, and by the Israel Ministry of Science

$e$ supported by the German-Israeli Foundation, the Israel Science Foundation, the U.S.-Israel Binational Science Foundation, and by the Israel Ministry of Science

$f \quad$ supported by the Italian National Institute for Nuclear Physics (INFN)

$g$ supported by the Japanese Ministry of Education, Science and Culture (the Monbusho) and its grants for Scientific Research

$h$ supported by the Korean Ministry of Education and Korea Science and Engineering Foundation

${ }^{i}$ supported by the Netherlands Foundation for Research on Matter (FOM)

$j$ supported by the Polish State Committee for Scientific Research, grant No. 115/E-343/SPUB/P03/002/97, 2P03B10512, 2P03B10612, 2P03B14212, 2P03B10412

$k$ supported by the Polish State Committee for Scientific Research (grant No. 2P03B08614) and Foundation for Polish-German Collaboration

$l$ partially supported by the German Federal Ministry for Education and Science, Research and Technology (BMBF)

$m$ supported by the Fund for Fundamental Research of Russian Ministry for Science and Education and by the German Federal Ministry for Education and Science, Research and Technology (BMBF)

$n$ supported by the Spanish Ministry of Education and Science through funds provided by CICYT

$o$ supported by the Particle Physics and Astronomy Research Council

$p$ supported by the US Department of Energy

$q$ supported by the US National Science Foundation 


\section{Introduction}

Perturbative QCD can be applied to ep scattering to calculate the amplitude for elastic production of heavy vector mesons. Previous HERA results on $J / \psi$ meson production at $Q^{2} \simeq 0$ and for $0.25<Q^{2}<40 \mathrm{GeV}^{2}$ [1], 2] have shown that the rise of the cross section with $W$ as well as the dependence of the cross section with $Q^{2}$ can be described by perturbative QCD models [3, 4 , 5]. Only upper limits of $\sigma \cdot \mathcal{B}$ exist in the literature for inclusive $\Upsilon$ production in lepton-hadron collisions [6].

In this paper we extend the study of elastic photoproduction of vector mesons at HERA to $\Upsilon$ mesons. The measurement is made with the ZEUS detector using a data sample which corresponds to an integrated ep luminosity of $43.2 \mathrm{pb}^{-1}$. The improved luminosity allows the study of the reaction $\gamma p \rightarrow \mu^{+} \mu^{-} p$ for $\mu^{+} \mu^{-}$invariant masses beyond the $\Upsilon$ mass region. A first measurement of $\sigma_{\gamma p \rightarrow \Upsilon p} \cdot \mathcal{B}$ in photoproduction is presented in the kinematic range of the photon-proton centre of mass energy $80<W<160 \mathrm{GeV}$, where $\mathcal{B}$ is the $\Upsilon$ branching ratio to muons. The $\Upsilon(1 S), \Upsilon(2 S)$ and $\Upsilon(3 S)$ resonances are not resolved in this measurement. Under the assumption that the production ratios of $\Upsilon(1 S)$, $\Upsilon(2 S)$ and $\Upsilon(3 S)$ are the same as those measured in hadron-hadron collisions [7, \&], we determine the $\Upsilon(1 S)$ and the ratio of the $\Upsilon(1 S)$ to $J / \psi$ photoproduction cross sections and compare them to a pQCD inspired model [5].

\section{Experimental Conditions}

In the years 1995-97 HERA collided positrons of $27.5 \mathrm{GeV}$ with protons of $820 \mathrm{GeV}$, corresponding to a centre of mass energy $\sqrt{s}=300 \mathrm{GeV}$. A description of the ZEUS detector can be found in references [9, 10]. The primary components used in this analysis were the central tracking detector, the uranium-scintillator calorimeter, the muon chambers and the proton remnant tagger. The central tracking detector (CTD) 11 operates in a $1.43 \mathrm{~T}$ solenoidal magnetic field. It is a drift chamber consisting of 72 cylindrical layers, organized into 9 superlayers. It was used to identify the vertex and to measure the momenta and directions of the muons. The transverse momentum resolution is $\sigma\left(p_{t}\right) / p_{t}=\left[0.005 p_{t}\right] \oplus 0.016$, with $p_{t}$ in $\mathrm{GeV}$, for full length tracks. The calorimeter (CAL) 12 is hermetic. Under test beam conditions, it has energy resolutions of $18 \% / \sqrt{E}$ for electrons and $35 \% / \sqrt{E}$ for hadrons. The time resolution is below $1 \mathrm{~ns}$ for energy deposits greater than $4.5 \mathrm{GeV}$. The CAL was used to reject cosmic rays and beam halo background by timing and to identify minimum ionizing particles (m.i.p.). It is surrounded by a magnetized iron yoke with a field of $1.4 \mathrm{~T}$ produced by conventional warm coils. The muon system (MUO) consists of tracking detectors (forward, barrel and rear muon chambers: FMU [10], B/RMU [13]) placed inside and outside the yoke covering the polar angles from $10^{\circ}$ to $171^{\circ}$. They were used for the trigger and to tag the muons by matching segments in the MUO chambers with tracks in the CTD and m.i.p.'s in the CAL. The proton remnant tagger (PRT) consists of two stations of scintillation counters surrounding the beamline at $Z=5 \mathrm{~m}$ and $Z=24 \mathrm{~m}$ 凹, and tags particles in the forward proton direction in an angular range 6 to 26

\footnotetext{
${ }^{1}$ The right-handed ZEUS coordinate system is centred on the nominal interaction point $(Z=0)$ and defined with the $Z$ axis pointing in the proton beam direction, and the horizontal $X$ axis pointing towards the centre of HERA.
} 
mrad and 1.5 to $8 \mathrm{mrad}$, respectively. It was used to estimate proton dissociative background. The luminosity was measured to a precision of $1.5 \%$ from the rate of energetic bremsstrahlung photons produced in the process $e^{+} p \rightarrow e^{+} \gamma p$.

\section{Kinematics}

Figure 11a shows a schematic diagram for the reaction

$$
e^{+}(k) p(p) \rightarrow e^{+}\left(k^{\prime}\right) V(v) p\left(p^{\prime}\right),
$$

where the symbol in parenthesis denotes the four-momentum of the corresponding particle, and $V$ indicates a $J / \psi$ or an $\Upsilon$.

The kinematics of the inclusive scattering of unpolarised positrons and protons are described by the positron-proton centre of mass energy squared $(s)$ and any two of the following variables:

- $Q^{2}=-q^{2}=-\left(k-k^{\prime}\right)^{2}$, the negative four-momentum squared of the exchanged photon;

- $y=(q \cdot p) /(k \cdot p)$, the fraction of the positron energy transferred to the hadronic final state in the rest frame of the initial state proton;

- $W^{2}=(q+p)^{2}=-Q^{2}+2 y(k \cdot p)+M_{p}^{2}$, the centre of mass energy squared of the photon-proton system, where $M_{p}$ is the proton mass.

For a complete description of the exclusive reaction $e^{+} p \rightarrow e^{+} V p\left(V \rightarrow \mu^{+} \mu^{-}\right)$the following additional variables are required:

- $M_{V}$, the invariant mass of the $\mu^{+} \mu^{-}$pair;

- $t=\left(p-p^{\prime}\right)^{2}$, the four-momentum transfer squared at the proton vertex;

- $\Phi$, the angle between the vector meson production plane and the positron scattering plane in the photon-proton centre of mass frame;

- $\theta_{h}$ and $\phi_{h}$, the polar and azimuthal angles of the positively charged decay lepton in the $V$ helicity frame.

In this analysis, photoproduction events are selected by requesting a $\mu^{+} \mu^{-}$pair from the interaction point and nothing else in either the CTD or the CAL. For the selected events the $Q^{2}$ value ranges from the kinematic minimum $Q_{\min }^{2}=M_{e}^{2} y^{2} /(1-y) \approx 10^{-9} \mathrm{GeV}^{2}$, where $M_{e}$ is the electron mass, to the value at which the scattered positron starts to be observed in the uranium calorimeter $Q_{\max }^{2} \approx 1 \mathrm{GeV}^{2}$, with a median $Q^{2}$ of approximately $5 \times 10^{-5} \mathrm{GeV}^{2}$. Since the typical $Q^{2}$ is small, the photon-proton centre of mass energy can be expressed as

$$
W^{2} \approx 4 E_{p} E_{e} y \approx 2 E_{p}\left(E-p_{Z}\right)_{V}
$$

where $E_{p}$ is the laboratory energy of the incoming proton and $\left(E-p_{Z}\right)_{V}$ is the difference between the energy and the longitudinal momentum of the vector meson, $V$, as determined from the CTD tracks assuming the muon mass. $\Phi$ is not measurable since the scattered positron is not detected. 


\section{Event Selection}

Elastic $\mu^{+} \mu^{-}$events were selected using dedicated triggers. Trigger cuts are superseded by the following offline cuts:

- CAL timing and reconstructed interaction vertex consistent with the nominal ep interaction to reject non $e^{+} p$ background;

- two oppositely charged tracks from the vertex, at least one of which matches a segment in the B/RMU chambers or a hit in the FMU chambers, and no other track in the CTD;

- at least 3 CTD superlayers per track, limiting the polar angular region from $\simeq 17^{\circ}$ to $\simeq 163^{\circ}$

- acollinearity of the tracks $\Omega<174^{\circ}$ where $\Omega$ is the angle between the two tracks, to reject cosmic ray events;

- invariant mass of the two CTD tracks, treated as muons, larger than $2 \mathrm{GeV}$;

- CAL energy associated to each track consistent with the energy deposit of a m.i.p.;

- no CAL cell (apart from those associated with a $\mu$ ) with energy greater than 150 $\mathrm{MeV}$, well above the CAL uranium noise level.

The events were then selected in the kinematical range of $W$ between 80 and $160 \mathrm{GeV}$, corresponding to an acceptance of $\approx 40 \%$ for the $\Upsilon$; the acceptance for the $J / \psi$ falls smoothly from $38 \%$ to $10 \%$ with increasing $W$ over this $W$ range.

\section{Monte Carlo Simulation}

To compute the acceptance, the reaction 1 (Figure 1 1a) was simulated using the DIPSI generator [14. DIPSI is based on a model developed by Ryskin [4] in which the exchanged photon fluctuates into a $q \bar{q}$ pair which interacts with a gluon ladder emitted by the incident proton. The parameters of the model are the strong coupling constant $\alpha_{s}$ (assumed fixed), the two-gluon form factor and the gluon momentum density of the proton. The cross section dependence on $W$ and $t$ is fixed by these parameters.

Proton dissociative vector meson production, $e^{+} p \rightarrow e^{+} V N$ (Figure 1]b), was simulated using the EPSOFT [15] and the DIFFVM [16] generators. Both are based on the assumption that the diffractive cross section at large $M_{N}$ is of the form $d^{2} \sigma / d|t| d M_{N}^{2} \propto e^{-b|t|} / M_{N}^{\beta}$ where $M_{N}$ is the mass of the dissociative system; we have used $b=1 \mathrm{GeV}^{-2}$ and $\beta=2.2$. The simulation of the dissociative system includes a parametrisation of the spectrum in the resonance region which differs for the two generators.

The background Bethe-Heitler process, in which a lepton pair is produced by the fusion of a photon radiated by the positron with a photon radiated by the proton, was simulated using the LPAIR generator [17]. Both elastic and proton dissociative events were generated. 
All Monte Carlo events were passed through a simulation of the ZEUS detector and trigger based on the GEANT program [18] and analysed with the same reconstruction and offline selection procedures as the data. The overall acceptance in a selected kinematic range was obtained as the ratio of the number of Monte Carlo events passing the cuts to the number of events generated in the same range. The acceptance, calculated in this manner, accounts for the geometric acceptance, for the detector, trigger and reconstruction efficiencies, and for the detector resolution.

\section{Analysis}

The measured $\mu^{+} \mu^{-}$mass distribution, for the sample of events obtained by the selection described in Section 1 , is shown in Figure 2. The signals of the $J / \psi, \psi(2 S)$ and $\Upsilon$ 's (unresolved) are apparent. The continuum is well described by the Bethe-Heitler process apart from an enhancement in the region of $6 \mathrm{GeV}$, which is consistent with being a fluctuation.

\subsection{Extraction of the Signal}

The elastic Bethe-Heitler process can be calculated to good precision since it is a pure QED reaction. There is, however, some arbitrariness in the parametrisation of the proton dissociative component. Comparing the data with the LPAIR simulation in the mass interval 4 to $8 \mathrm{GeV}$, where we expect the Bethe-Heitler process to dominate, we find that the simulation underestimates the energy deposited in the forward region of CAL due to proton dissociation. This leads to an overestimate of the Bethe-Heitler events which pass the selection cuts if both the elastic and the proton dissociative processes are normalised to luminosity by their calculated cross sections [17]. However, since the shape of the $\mu^{+} \mu^{-}$mass distribution predicted by LPAIR is the same for both processes, to evaluate this background we have normalised Monte Carlo and data in a mass window not containing resonances $(4.2$ to $8.4 \mathrm{GeV})$. This corresponds to adding a $10 \%$ contribution to the elastic Bethe-Heitler distribution normalised to the luminosity. In Figure 2 the data are compared with this renormalised Bethe-Heitler distribution. The spectrum outside the resonance regions is well reproduced and the distribution has been used to subtract the background under the resonances.

The limited statistics and the $\mu^{+} \mu^{-}$mass resolution of $0.3 \mathrm{GeV}$ in the $\Upsilon$ region do not allow to distinguish between the $\Upsilon(1 S), \Upsilon(2 S)$ and $\Upsilon(3 S)$ states. The mass window 8.9 to $10.9 \mathrm{GeV}$ (i.e. from twice the resolution below the $\Upsilon(1 \mathrm{~S})$ nominal mass, $9.46 \mathrm{GeV}$, to twice the resolution above the $\Upsilon(3 \mathrm{~S})$ mass, $10.36 \mathrm{GeV}$ 19]) was chosen to count the $\Upsilon$ events. In this region there are 57 events while 39.9 are estimated to be background. The mass spectrum in the $\Upsilon$ region after background subtraction is shown in the insert of Figure 2; the mean mass and the r.m.s. for the background corrected signal in the window are $9.9 \pm 0.2 \mathrm{GeV}$ and $0.47 \mathrm{GeV}$, respectively. The simulation of the detector response to a mixture of $\Upsilon$ states [7] yields a mean mass of $9.7 \mathrm{GeV}$ and an r.m.s. of 0.42 $\mathrm{GeV}$. The r.m.s. is smaller since background was not considered. A total of 4257 events are counted in the $J / \psi$ mass window from 2.8 to $3.35 \mathrm{GeV}$ while 306 are expected from the Bethe-Heitler process. 
The amount of proton dissociative resonant background remaining in the sample after the cuts described in Section 1 is estimated using the number of events tagged by the PRT and the EPSOFT Monte Carlo to correct for the detector acceptance. In the $J / \psi$ mass region the fraction of dissociative events is determined to be $25 \pm 2$ (stat.)\% . For $\Upsilon$ production, the same fraction of proton dissociative events has been assumed. Within the limited statistics, this assumption is consistent with the number of events in the $\Upsilon$ mass region tagged by the PRT.

\subsection{Systematic Uncertainties}

A study of the systematic uncertainties on the measurements has been performed. The systematic uncertainties, listed below, have been divided into two classes, those common to the $\Upsilon$ and $J / \psi$ analysis, which cancel in the measurement of the ratio, and those remaining.

- Common systematic uncertainties:

- uncertainty on the CTD first level trigger efficiency, $\pm 5 \%$;

- uncertainty on the muon chamber and muon trigger efficiency, $\pm 10 \%$;

- uncertainty on the proton dissociation background estimated using DIFFVM, $(+9,-2) \%$.

- Systematic uncertainties specific to each meson:

- using a different mass region for normalisation of background contribution, from 4 to $6 \mathrm{GeV},+10 \%$;

- varying the $\Upsilon$ mass window by $\pm 300 \mathrm{MeV}, \pm 10 \%$;

- varying the $\cos \theta_{h}$ distribution used in the Monte Carlo from that required by s-channel helicity conservation $\left(1+\cos ^{2} \theta_{h}\right)$ to a flat distribution for the $\Upsilon$ and between the limits allowed by the existing measurements for the $J / \psi,-8 \%$ for the $\Upsilon,-4 \%$ for the $J / \psi$;

- the uncertainties on the $J / \psi$ and $\Upsilon$ muonic branching ratios, $\pm 3 \%$.

All of these uncertainties are added in quadrature yielding an overall systematic error on $\sigma \cdot \mathcal{B}$ of $(+20,-17) \%$. The systematics which affect the $\sigma_{\gamma p \rightarrow \Upsilon_{p}} / \sigma_{\gamma p \rightarrow J / \psi p}$ ratio are only those included in the second group which give contributions of $(+14,-13) \%$.

Other possible sources of systematic uncertainty (variation of the energy thresholds used to select the elastic events, removal of the m.i.p. requirement on the energy associated with the tracks, variation of the $J / \psi$ mass window, uncertainty in the luminosity determination, variation of the DIPSI parameters, use of an unbinned fit to extract the $\Upsilon$ signal) give negligible contributions. 


\section{Results and comparison with QCD predictions}

We have calculated $\sigma \cdot \mathcal{B}$ as

$$
\sigma_{e^{+} p \rightarrow e^{+} V p} \cdot \mathcal{B}=\frac{N_{e v t}(1-\mathcal{F})}{\mathcal{A} \mathcal{L}}
$$

where $N_{\text {evt }}$ denotes the background subtracted number of $V$ signal events, $\mathcal{F}$ is the estimated fraction of proton dissociative events, $\mathcal{A}$ the acceptance, $\mathcal{L}$ the integrated luminosity and $\mathcal{B}$ the muonic branching fraction. Photoproduction cross sections were determined by dividing the electroproduction cross sections by the photon flux, calculated according to reference [20].

The production cross sections multiplied by the muonic branching ratios for the unresolved $\Upsilon(1 S), \Upsilon(2 S)$ and $\Upsilon(3 S)$ are summarized in Table 11. $\sigma_{\gamma p \rightarrow \Upsilon_{p}} \cdot \mathcal{B}$ is determined to be $13.3 \pm 6.0$ (stat. $)_{-2.3}^{+2.7}$ (syst.) pb at a mean photon-proton centre of mass energy $\langle\mathrm{W}\rangle=120$ $\mathrm{GeV}$. The first error is statistical and the second is the systematic uncertainty. In Table 1 we report also the ratio $\sigma_{\gamma p \rightarrow \Upsilon p} \cdot \mathcal{B}$ summed on the $\Upsilon$ states over $\sigma_{\gamma p \rightarrow J / \psi p}$ in the $W$ range between 80 and $160 \mathrm{GeV}$.

The QCD-based model of reference [5] gives predictions for the hard diffractive photo- and electroproduction of heavy vector meson $J / \psi$ and $\Upsilon(1 S)$ within the leading logarithmic approximation. To compare the data with the predictions, the experimental cross section for $\Upsilon(1 S)$ has to be derived. As our data does not allow the relative fractions of $\Upsilon(1 S)$, $\Upsilon(2 S)$ and $\Upsilon(3 S)$ to be determined, we have to make assumptions on their relative production rates. Assuming that the cross sections times branching ratios are the same as measured by $\mathrm{CDF}$ [7], $\sigma \cdot \mathcal{B}(\Upsilon(2 S)) / \sigma \cdot \mathcal{B}(\Upsilon(1 S))=0.281 \pm 0.030($ stat. $) \pm 0.038($ sys $)$ and $\sigma \cdot \mathcal{B}(\Upsilon(3 S)) / \sigma \cdot \mathcal{B}(\Upsilon(1 S))=0.155 \pm 0.024$ (stat) \pm 0.021 (sys), the $\Upsilon(1 S)$ accounts for $70 \%$ of the signal. Using the muonic branching ratio $\mathcal{B}\left(\Upsilon(1 S) \rightarrow \mu^{+} \mu^{-}\right)=(2.48 \pm 0.007) \%$ [19], we derive

$$
\sigma_{\gamma p \rightarrow \Upsilon(1 S) p}=375 \pm 170(\text { stat. })_{-64}^{+75}(\text { syst. }) \text { pb at } W=120 \mathrm{GeV}
$$

and the ratio

$$
\sigma_{\gamma p \rightarrow \Upsilon(1 S) p} / \sigma_{\gamma p \rightarrow J / \psi p}=\left(4.8 \pm 2.2(\text { stat. })_{-0.6}^{+0.7}(\text { syst. })\right) \times 10^{-3} .
$$

In Figure 3(a),(b) these values are compared with the theoretical calculations which give

$\approx 60 \mathrm{pb}$ for $\sigma_{\gamma p \rightarrow \Upsilon(1 S) p}$ and $\approx 0.001$ for the ratio, both weakly dependent on the structure function parametrisation used. Our measurement is higher than the predictions.

\section{Acknowledgments}

We thank the DESY Directorate for their strong support and encouragement. The experiment was made possible by the inventiveness and the diligent efforts of the DESY machine group. The design, construction and installation of the ZEUS detector have been made possible by the ingenuity and dedicated efforts of many people from inside 
DESY and from the home institutes who are not listed as authors. Their contributions are acknowledged with great appreciation.

We thank L. Frankfurt and M. Strikman for valuable discussions and R. Engel and M. McDermott for making their tables of theoretical predictions available. 


\section{References}

[1] ZEUS Collab., J. Breitweg et al., Z. Phys. C75 (1997) 215.

[2] H1 Collab., S. Aid et al., Nucl. Phys. B472 (1996) 3;

H1 Collab., S. Aid et al., Nucl. Phys. B468 (1996) 3.

[3] S.J. Brodsky et al., Phys. Rev. D50 (1994) 3134.

[4] M.G. Ryskin, Z. Phys. C57 (1993) 89;

M.G. Ryskin, R.G. Roberts, A.D. Martin and E.M. Levin, Z. Phys. C76 (1997) 231.

[5] L. Frankfurt, W. Koepf and M. Strikman, Phys. Rev. D57 (1998) 512;

R. Engel and M. McDermott, private communication.

[6] BCDMS Collab., D. Bollini et al., Nucl. Phys. B199 (1982) 27;

EMC Collab., J.J. Aubert et al., Nucl. Phys. B213 (1983) 1.

[7] CDF Collab., F. Abe et al., Phys. Rev. Lett. 75 (1995) 4358.

[8] J.H. Cobb et al., Phys. Lett. B72 (1977) 273;

K. Ueno et al., Phys. Rev. Lett. 42 (1979) 486;

J. Badier et al., Phys. Lett. B86 (1979) 98;

C. Kourkoumelis et al., Phys. Lett. B91 (1980) 481;

NA10 Collab., S. Falciano et al., Phys. Lett. B158 (1985) 92;

NA10 Collab., M. Grossmann-Handschin et al., Phys. Lett. B179 (1986) 170;

E605 Collab., T. Yoshida et al., Phys. Rev. D39 (1989) 3516;

G. Moreno et al., Phys. Rev. D43 (1991) 2815;

E771 Collab., T. Alexopoulos et al., Phys. Lett. B374 (1996) 271.

[9] ZEUS Collab., M. Derrick et al., Phys. Lett. B293 (1992) 465;

ZEUS Collab., M. Derrick et al., Z. Phys. C63 (1994) 391.

[10] ZEUS Collab., The ZEUS Detector, Status Report 1993, DESY 1993.

[11] N. Harnew et al., Nucl. Inst. Meth. A279 (1989) 290;

B. Foster et al., Nucl. Phys. B (Proc. Suppl.) 32 (1993) 181;

B. Foster et al., Nucl. Inst. Meth. A338 (1994) 254.

[12] M. Derrick et al., Nucl. Inst. Meth. A309 (1991) 77;

A. Andresen et al., Nucl. Inst. Meth. A309 (1991) 101;

A. Bernstein et al., Nucl. Inst. Meth. A336 (1993) 23.

[13] G. Abbiendi et al., Nucl. Inst. Meth. A333 (1993) 342.

[14] M. Arneodo, L. Lamberti and M.G. Ryskin, Comp. Phys. Comm. 100 (1997) 195.

[15] M. Kasprzak, PhD Thesis, Warsaw University, DESY F35D-96-16 (1994).

[16] B. List, Diploma Thesis, Techn. Univ. Berlin (1993) unpublished. 
[17] J.A.M. Vermaseren, Nucl. Phys., B229 (1983) 347;

S.P. Baranov, O. Dünger, H. Shooshtari and J.A.M. Vermaseren, Proc. of the Workshop 'Physics at HERA', Vol. III, Oct. 1991, 1478.

[18] GEANT 3.13: R. Brun et al., CERN DD/EE-84-1 (1987).

[19] Review of Particle Properties, Particle Data Group, R.M. Barnett et al., Phys. Rev. D54 (1996) 1.

[20] V.N. Gribov, V.A. Kolkunov, L.B. Okun and V.M. Shekhter, Sov. Phys. JETP 14 (1962) 1308;

V.M. Budnev, I.F. Ginzburg, G.V. Meledin and V.G. Serbo, Phys. Rep. C15 (1975) 181. 


\begin{tabular}{|c|c|c|c|c|c|c|c|}
\hline $\begin{array}{c}W \text { range } \\
(\mathrm{GeV})\end{array}$ & $\begin{array}{c}\langle W\rangle \\
(\mathrm{GeV})\end{array}$ & $N_{\text {evt }}$ & $\mathcal{A}$ & $\begin{array}{c}\sigma_{e p \rightarrow e \Upsilon p} \cdot \mathcal{B} \\
(\mathrm{pb})\end{array}$ & $\Phi_{T}$ & $\begin{array}{c}\sigma_{\gamma p \rightarrow \Upsilon p} \cdot \mathcal{B} \\
(\mathrm{pb})\end{array}$ & $\frac{\sigma_{\gamma p \rightarrow \Upsilon_{p}} \cdot \mathcal{B}}{\sigma_{\gamma p \rightarrow J / \psi p}} \times 10^{4}$ \\
\hline $80-160$ & 120 & $17.1 \pm 7.5$ & 0.43 & $0.68 \pm 0.30_{-0.12}^{+0.14}$ & 0.051 & $13.3 \pm 6.0_{-2.3}^{+2.7}$ & $1.7 \pm 0.8_{-0.2}^{+0.2}$ \\
\hline
\end{tabular}

Table 1: The results for the unresolved $\Upsilon$ cross sections times the muonic branching ratios, $\sum_{i} \sigma_{i} \cdot \mathcal{B}_{i}$, where $i$ runs over the three states $\Upsilon(1 S), \Upsilon(2 S), \Upsilon(3 S)$ and $\mathcal{B}_{i}$ is the branching ratio of each state to muons. $N_{\text {evt }}$ is the number of events after subtraction of the non-resonant background contribution: $75 \%$ of $N_{\text {evt }}$ is attributed to the elastic reaction, the remaining $25 \%$ to the proton dissociative process. $\mathcal{A}$ is the acceptance and $\Phi_{T}$ is the photon flux used to calculate the $\gamma p$ cross section $\sigma_{\gamma p \rightarrow \Upsilon p}$ from the $e p$ cross section $\sigma_{e p \rightarrow e \Upsilon p}$. The last column contains the ratio of unresolved $\Upsilon$ cross section times the muonic branching fraction to $J / \psi$ cross section. The first uncertainties are statistical and the second ones systematic. 

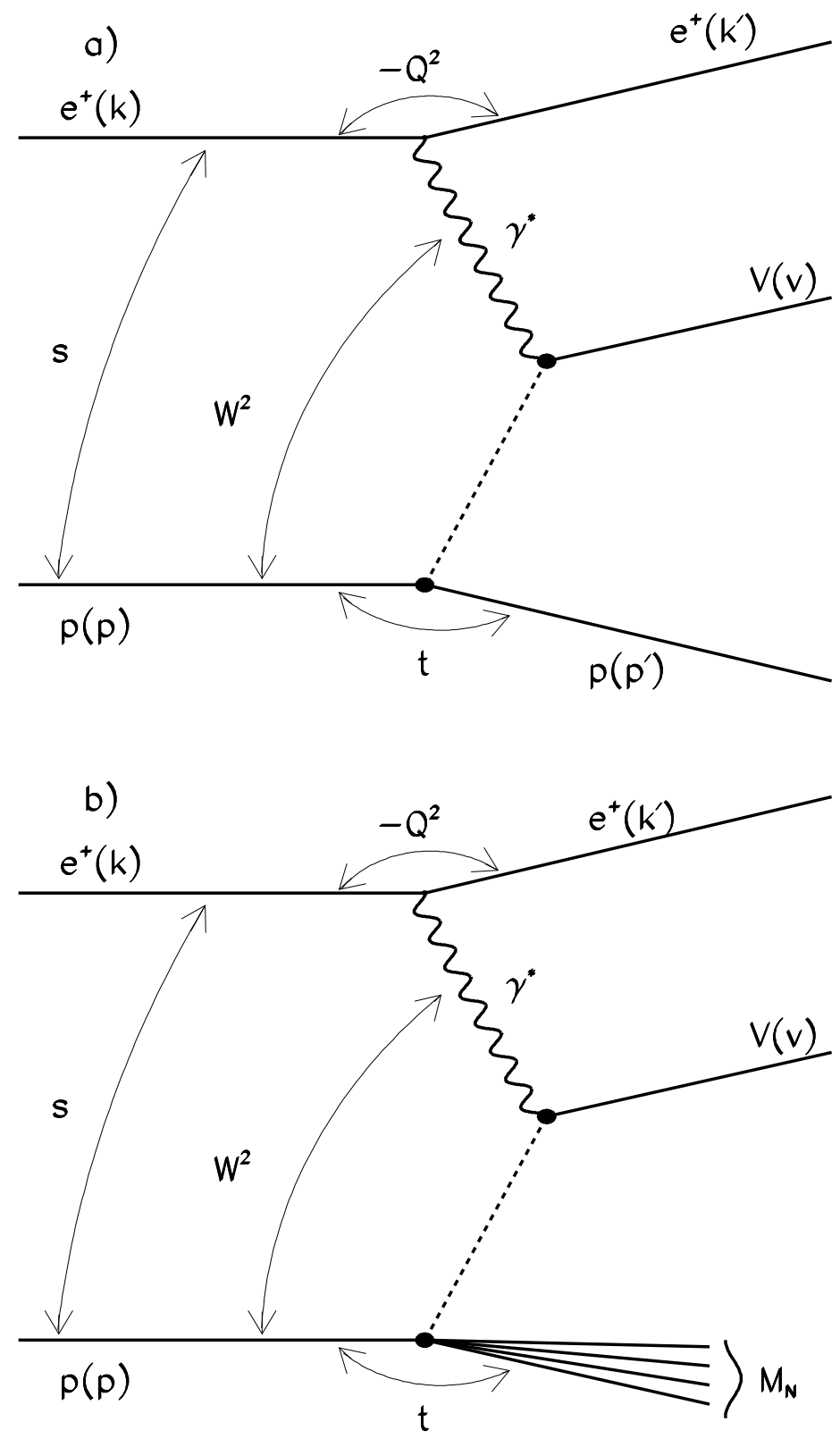

Figure 1: Schematic diagrams for diffractive vector meson $(V)$ electroproduction. (a) Elastic $V$ production. (b) Proton dissociative $V$ production where the proton dissociates into a hadronic system of invariant mass $M_{N}$. The dotted line represents a colorless exchange between the virtual photon and the proton. 


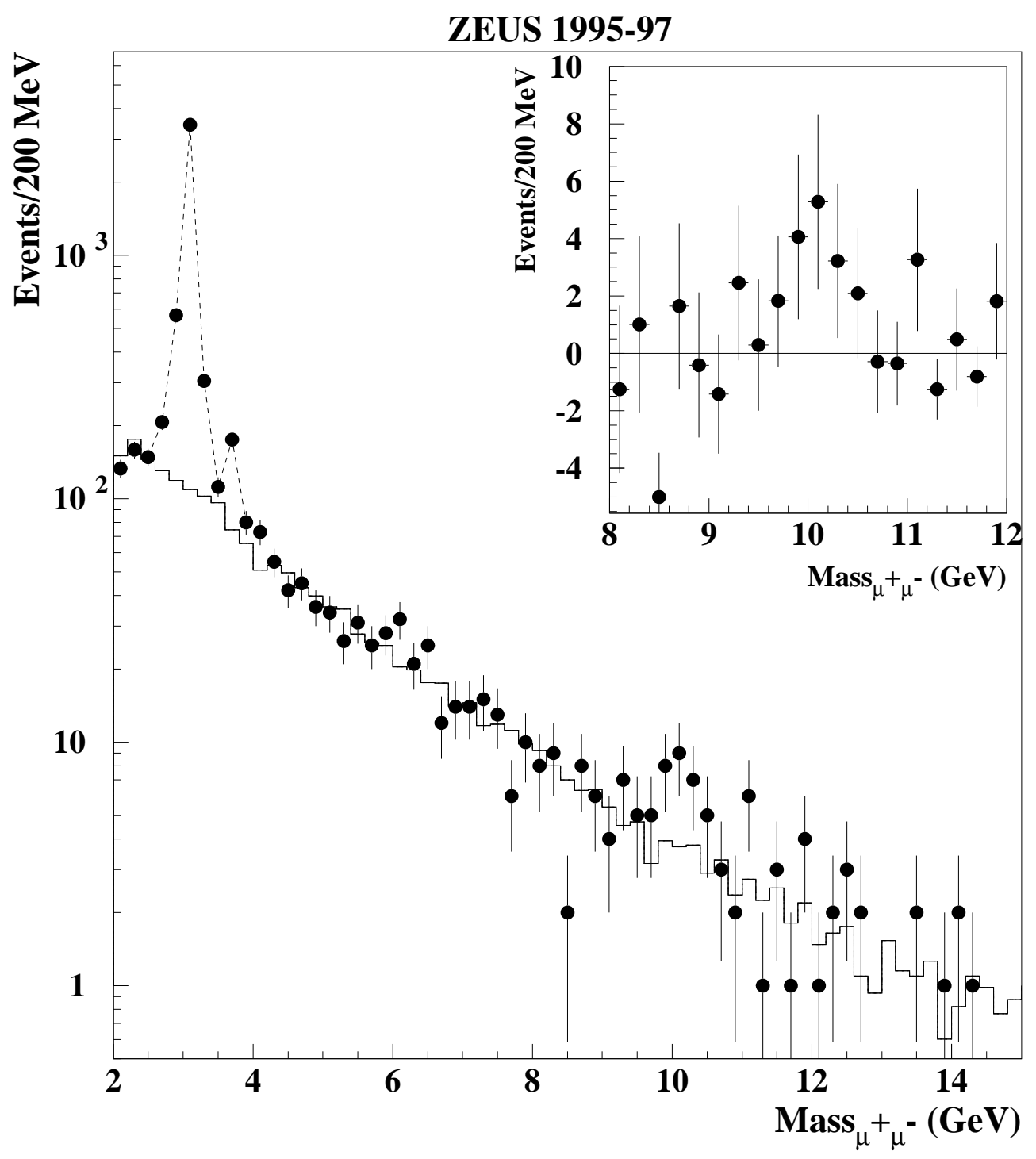

Figure 2: Mass distribution of $\mu^{+} \mu^{-}$pairs. The histogram represents the simulated Bethe-Heitler background. Points in the $J / \psi$ region are connected by a dotted line to guide the eye. The insert shows the signal remaining in the $\Upsilon$ region after subtraction of the non-resonant background. 

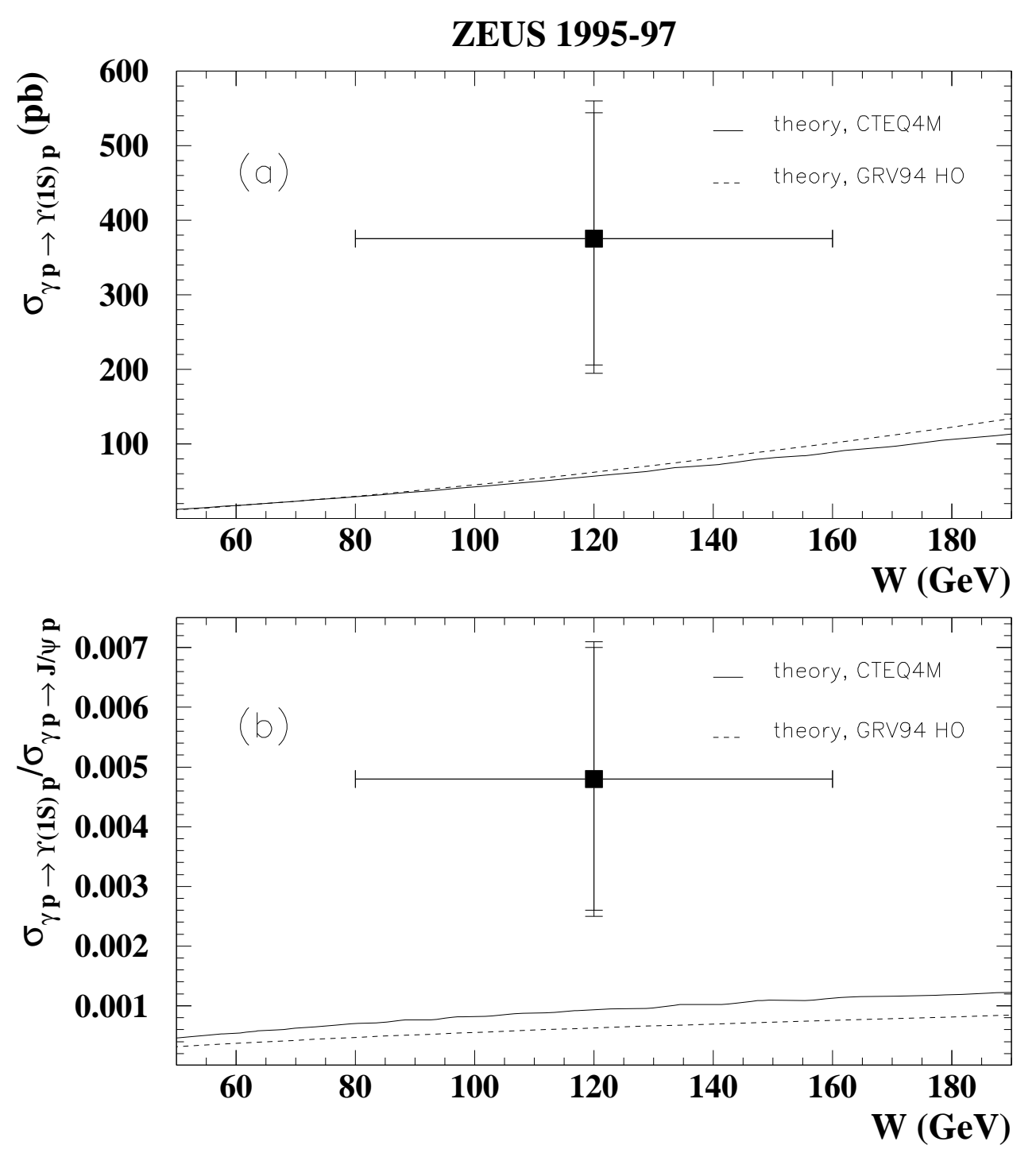

Figure 3: (a) $\sigma_{\gamma p \rightarrow \Upsilon(1 S) p}$ and (b) the ratio $\sigma_{\gamma p \rightarrow \Upsilon(1 S) p} / \sigma_{\gamma p \rightarrow J / \Psi p}$ as a function of $W$, the centre of mass energy of the photon-proton system. Data (full squares) are compared with the predictions of [5] for the GRV94(HO) (dashed line) and CTEQ(4M) (full line) parametrisations of the proton structure function. The data have been scaled taking into account the muonic branching ratio and the contributions of the $\Upsilon(2 \mathrm{~S})$ and $\Upsilon(3 \mathrm{~S})$, as described in the text. The inner error bars show the statistical errors, the outer bars correspond to the statistical and systematic errors added in quadrature. 